\title{
EVALUACIÓN DE LAS PARTÍCULAS EN SUSPENSIÓN ATMOSFÉRICA Y BIOAEROSOLES FÚNGICOS EN PARQUES URBANOS DE LA CIUDAD DE SANTO DOMINGO, REPÚBLICA DOMINICANA
}

\section{Evaluation of atmospheric suspended particles and fungal bioaerosols in urban parks in the city of Santo Domingo, Dominican Republic}

\section{Andri Jesús Binet Álvarez \\ Profesor Ayudante de la Universidad Autónoma de \\ Santo Domingo, República Dominicana \\ ORCID: 0000-0003-2638-1406 \\ Correo-e: abinet63@uasd.edu.do}

\author{
Ángel Guillemes Peira \\ Profesor Fondo Verde Internacional, España \\ ORCID: 0000-0002-1037-3375 \\ Correo-e: angel.guillemes@fondoverde.org
}

Recibido: 28/4/2020 • Aprobado: 15/6/2020

Cómo citar: Binet Álvarez, A. J., \& Peira, Ángel G. (2020). Evaluación de las partículas en suspensión atmosférica y bioaerosoles fúngicos en parques urbanos de la ciudad de Santo Domingo, República Dominicana. Ciencia, Ambiente y Clima, 3(1), 47-54. Doi: https://doi.org/10.22206/cac.2020.v3i1.pp47-54

\section{Resumen}

Este estudio determinó el nivel de partículas en suspensión con diámetros $\leq 10 \mu \mathrm{m}$ e $\leq 2,5 \mu \mathrm{m}$, y oportunistas fúngicos asociados en los parques urbanos de Mirador Sur, Iberoamericano y Colón, de la ciudad de Santo Domingo en la República Dominicana. Para esto se realizaron dos muestreos con intervalo de 24 horas, utilizando un muestreador PCE-MPC 10 para cuantificar el particulado y un Kestrel 5500 para variables atmosféricas. Las esporas fueron colectadas mediante la técnica de sedimentación en placa con agar Sabouraud e identificadas utilizando un microscopio Olimpus CX33. Según los resultados en ambos muestreos, el nivel del particulado de rango PM $2.5 \mu \mathrm{m}$ y $10 \mu \mathrm{m}$ en los parques no supera el límite presente en el Reglamento Técnico Ambiental de Calidad del Aire del Ministerio de Medio Ambiente y Recursos Naturales de la República Dominicana. Entre los hongos identificados se encuentran: Alternaria alternata, Aspergillus sp. 1, Aspergillus niger, Cladosporium, Dreschlera, Penicillium sp. 1, P. sp. 2, Paecilomyces sp., Nattrasia sp., Fusarium sp., Rhizophus sp., y Ulocladium $s p$.

Palabras clave: particulado; bioaerosoles; hongos; contaminación atmosférica; parques urbanos.

\begin{abstract}
This study determined the level of suspended particles with diameters $\leq 10 \mu \mathrm{m}$ and $\leq 2.5 \mu \mathrm{m}$, and associated fungal opportunists in the urban parks of Mirador Sur, Iberoamericano and Colón in the city of Santo Domingo in the Dominican Republic. For this purpose, two samples were taken with a 24-hour interval, using a PCE-MPC 10 sampler to quantify the particulate and a Kestrel 5500 for atmospheric variables. Spores were collected by means of the Sabouraud agar plate sedimentation technique and identified using an Olimpus CX33 microscope. According to the results of both samples, the level of the PM 2.5 $\mu \mathrm{m}$ and $10 \mu \mathrm{m}$ range particles in the parks does not exceed the limit present in the Environmental Technical Regulation of Air Quality of the Ministry of Environment and Natural Resources of the Dominican Republic. Among the fungi identified are Alternaria alternata, Aspergillus sp. 1, Aspergillus niger, Cladosporium, Dreschlera, Penicillium sp. 1, P. sp. 2, and Paecilomyces sp., Nattrasia sp., Fusarium sp., Rhizophus sp., and Ulocladium sp.
\end{abstract}

Keywords: Particulate; bioaerosols; fungi; air pollution; urban parks. 


\section{Introducción}

La contaminación atmosférica por material particulado en suspensión es uno de los efectos ocasionados por la creciente urbanización e industrialización, lo cual afecta la calidad del aire, considerado un problema de salud pública en progresivo aumento, tanto a nivel global como regional (Montaño y Sandoval, 2007). El material en suspensión particulado atmosférico (PM) está integrado por partículas sólidas y/o líquidas (excepto el agua pura) suspendidas en la atmósfera (Suárez y Augusto, 2012), tiene como origen una gran variedad de fuentes naturales o antropogénicas y posee un amplio rango de propiedades morfológicas, físicas, químicas y termodinámicas.

Por su parte, el tamaño del PM es, posiblemente, un criterio fundamental que permite describir su comportamiento; la definición de un aerosol responde a que las partículas deben estar suspendidas en un gas, esto implica que las partículas son lo suficientemente pequeñas como para permanecer suspendidas por un determinado tiempo. De manera convencional, el límite de tamaño superior se considera que está cerca de $100 \mu \mathrm{m}$, estas partículas tienen una velocidad de sedimentación de $0.25 \mathrm{~m} / \mathrm{s}$. En este enorme intervalo de límites de tamańo que abarca hasta cuatro órdenes de magnitud, existen aerosoles con propiedades y comportamiento muy diferentes (Baron y Willeke, 2001). Saber la composición del PM brinda la posibilidad de conocer la calidad del aire que se respira en la zona (Quijano, Quijano y Henao, 2010). En torno a esto, en el 2005, la Organización Mundial de la Salud consideraba que el aire limpio es un requisito básico para la salud y el bienestar humano.

En cuanto al comportamiento de los hongos en el aire, son varios los trabajos existentes (Karim, 2010; López, 2012) que demuestran la ventaja selectiva que tienen en torno a la capacidad de liberar, dispersar y diseminar sus esporas y, ocasionalmente, fragmentos de hifas o conidióforos parciales en el aire para su dispersión (Kendrick, 2001). Las esporas pueden variar entre 2 a $500 \mu \mathrm{m}$, aunque en promedio es menor a $20 \mu \mathrm{m}$ (Estrada, Alvarado, Gutiérrez, Salcedo y Moreno, 2013).

La concentración de esporas de hongos en el aire varía según la ubicación, la altitud, la hora del día, la estación del año, el paisaje, el uso de la tierra, la condición de las áreas circundantes y las condiciones climáticas (Gioulekas et al., 2004; Mitakakis, O’Meara y Tovey, 2005). Determinar la distribución de esporas fúngicas en cualquier región puede ser útil para identificar asociaciones entre la sensibilidad fúngica y la prevención clínica de enfermedades alérgicas estacionales (Hedayati, Pasqualotto, Warn, Bowyer y Denning, 2007; Griffin, 2007).

\section{Materiales y métodos}

Se seleccionaron tres parques urbanos del Distrito Nacional de la República Dominicana para evaluar la calidad del aire. El primero es el parque Iberoamericano, considerado el antiguo parque Zoológico Nacional. Se encuentra en el centro de la ciudad de Santo Domingo, entre las Avenidas Pedro Henríquez Ureña y Simón Bolívar, y ocupa un espacio de $1.2 \mathrm{~km}^{2}$ aproximadamente, en las coordenadas $18^{\circ} 28^{\prime} 01^{\prime \prime} \mathrm{N}$ y $69^{\circ} 55^{\prime} 06^{\prime \prime} \mathrm{W}$. El parque posee un complejo de múltiples cavernas (Jiménez-Orozco, Lebrón-Liriano, Fernández-Gutiérrez, Urbáez y Guerrero, 2019). El segundo corresponde al parque Colón, se encuentran cercano al centro de la Zona Colonial entre las Calle Isabel La Católica, Arzobispo Meriño y Calle el Conde, a unos 250 metros del Río Ozama y de la zona portuaria. Cuenta con un perímetro aproximado de 313 metros en las coordenadas $18^{\circ} 28^{\prime} 24^{\prime \prime} \mathrm{N}$ y $69^{\circ} 53^{\prime} 01^{\prime \prime} \mathrm{W}$. El tercero, Mirador Sur; considerado el primer parque ecológico de Santo Domingo, se encuentra entre la Avenida de la Salud y Avenida Anacaona, adyacente a la Avenida Italia y Luperón. Es un parque urbano con un área superficial de $6 \mathrm{~km}^{2}$ aproximadamente, ubicándose en las coordenadas $18^{\circ} 26^{\prime} 31^{\prime \prime} \mathrm{N}$ y $69^{\circ} 57^{\prime} 32^{\prime \prime} \mathrm{W}$ (USAID-ICMA-FEDOMU-ADN, 2017). 


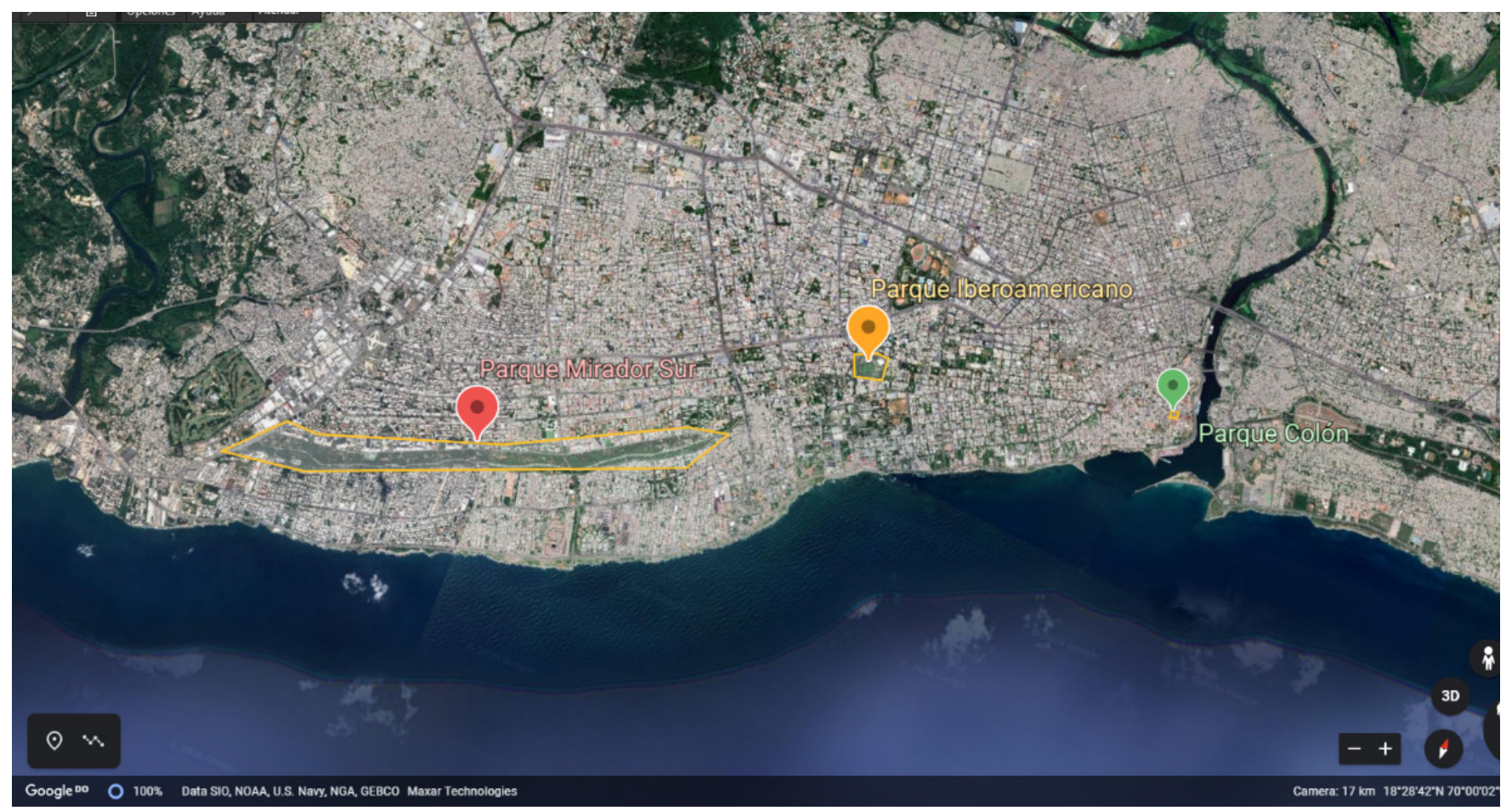

Figura 1. Ubicación de los parques Mirador Sur, Iberoamericano y Colón Fuente: elaboración propia.

\section{Obtención de muestras}

El muestreo y análisis de aire para determinar la calidad del particulado en suspensión y de los bioaerosoles fúngicos se efectuó de acuerdo con las normas metodológicas descritas por el Reglamento Técnico Ambiental de Calidad del Aire, del Ministerio de Medio Ambiente y Recursos Naturales de la República Dominicana.

Tabla 1. Estándares criterio de PM $10 \mu \mathrm{m}$ y $2,5 \mu \mathrm{m}$ en República Dominicana en tiempo promedio de 24 horas

\begin{tabular}{|l|c|c|}
\hline $\begin{array}{c}\text { Contaminante } \\
\text { criterio }\end{array}$ & Tiempo promedio & $\begin{array}{c}\text { Límite permisible } \\
(\mu \mathrm{g} / \mathbf{N m} 3)\end{array}$ \\
\hline $\begin{array}{l}\text { Partículas fracción } \\
(\mathrm{PM}-10)\end{array}$ & 24 horas & 150 \\
\hline $\begin{array}{l}\text { Partículas fracción } \\
(\mathrm{PM}-2,5)\end{array}$ & 24 horas & 65 \\
\hline
\end{tabular}

Fuente: elaboración propia.
El protocolo utilizado para la toma de muestras se ejecutó de la siguiente manera: se realizaron muestreos diurnos y nocturnos en las entradas y los puntos de mayor flujo de personas de los distintos parques, utilizando un muestreador de aire PCE-MPC 10. El muestreador de aire PCE-MPC 10 permite medir la concentración de partículas de forma rápida. En cuanto a los valores de las variables atmosféricas (temperatura y humedad relativa), se utilizó un Kestrel 5500. Para captar las esporas fúngicas se utilizó el método de sedimentación en placa de Petri con agar Sabouraud. Se realizaron muestreos por asentamiento en placas en momentos diferentes. Muestreos matinales entre las 9 y 11 a. m., y muestreos por la tarde entre las 3 y 8 p. m. La exposición de muestreo fue de unos 15 minutos de duración, utilizando las placas de Petri (9 $\mathrm{mm}$ de diámetro) con Sabouraud con eritromicina, para impedir el crecimiento bacteriano. Las placas fueron colocadas a unos 20 centímetros del suelo. 


\section{Observaciones morfológicas fúngicas}

Los organismos aislados fueron incubados durante tres días a una temperatura de $30{ }^{\circ} \mathrm{C}$. La identificación de los hongos fue llevada a cabo considerando la textura o aspecto, color, velocidad de crecimiento radial, tamaño $(\mathrm{mm})$, pigmentación del micelio y al anverso del medio, forma, borde y elevación de la colonia. La identificación de los géneros y especies fueron identificadas con base en Atlas of Airborne Fungal Spores in Europe (1993) y Samson, Hoekstra y Frisvad (2004), utilizando un Microscopio Olimpus CX33.

\section{Resultados y discusión}

Las tablas 2 y 3 ofrecen los niveles de partículas en suspensión con diámetro $\leq 10 \mu \mathrm{m} \mathrm{e} \leq 2,5 \mu \mathrm{m}$ y las condiciones climáticas de temperatura y humedad relativa en los días de muestreo en cada uno de los parques, respectivamente.

A continuación, en la tabla 2, se presentan los resultados del muestreo del particulado del primer día.

Tabla 2. Niveles de las partículas en suspensión con diámetro $\leq 10 \mu \mathrm{m}$ e $\leq 2,5 \mu \mathrm{m}$, temperatura y humedad relativa en los parques urbanos, Iberoamericano, Colón y Mirador Sur en el primer muestreo

\begin{tabular}{|c|c|c|c|c|c|c|}
\hline Parque & Hora & Coordenadas & PM $10 \mu \mathrm{m}$ & PM 2,5 $\mu \mathrm{m}$ & Temperatura ${ }^{\circ} \mathrm{C}$ & Humedad relativa $\%$ \\
\hline \multirow[t]{3}{*}{ Iberoamericano } & $18: 32$ & $18^{\circ} 28^{\prime} 03^{\prime \prime} \mathrm{N} 69^{\circ} 55^{\prime} 09^{\prime \prime} \mathrm{W}$ & 13 & 10 & 30 & 75 \\
\hline & $18: 35$ & $18^{\circ} 27^{\prime} 56^{\prime \prime} \mathrm{N} 69^{\circ} 55^{\prime} 07^{\prime \prime} \mathrm{W}$ & 8 & 4 & 29 & 73 \\
\hline & $18: 41$ & $18^{\circ} 27^{\prime} 57^{\prime \prime} \mathrm{N} 69^{\circ} 55^{\prime} 03^{\prime \prime} \mathrm{W}$ & 7 & 7 & 29 & 68 \\
\hline Colón & $19: 28$ & $18^{\circ} 28^{\prime} 24^{\prime \prime} \mathrm{N} \mathrm{69} 53^{\prime} 01^{\prime \prime} \mathrm{W}$ & 17 & 16 & 29 & 69 \\
\hline \multirow[t]{2}{*}{ Mirador Sur } & $10: 11$ & $18^{\circ} 27^{\prime} 00^{\prime \prime} \mathrm{N} 69^{\circ} 56^{\prime} 20^{\prime \prime} \mathrm{W}$ & 3 & 2 & 31 & 59 \\
\hline & 11: 02 & $18^{\circ} 26^{\prime} 31^{\prime \prime} \mathrm{N} 69^{\circ} 57^{\prime} 32^{\prime \prime} \mathrm{W}$ & 7 & 5 & 31 & 58 \\
\hline
\end{tabular}

Fuente: elaboración propia.

En el segundo muestreo las variables presentaron diferencia poco significativa en los tres parques (tabla 3).

Tabla 3. Niveles de las partículas en suspensión con diámetro $\leq 10 \mu \mathrm{m}$ e $\leq 2,5 \mu \mathrm{m}$, temperatura y humedad relativa en los parques urbanos Iberoamericano, Colón y Mirador Sur en el segundo muestreo

\begin{tabular}{|c|c|c|c|c|c|c|}
\hline Parque & Hora & Coordenadas & PM $10 \mu \mathrm{m}$ & PM 2,5 $\mu \mathrm{m}$ & Temperatura ${ }^{\circ} \mathrm{C}$ & Humedad relativa $\%$ \\
\hline \multirow[t]{3}{*}{ Iberoamericano } & $16: 37$ & $18^{\circ} 28^{\prime} 03^{\prime \prime} \mathrm{N} 69^{\circ} 55^{\prime} 09^{\prime \prime} \mathrm{W}$ & 4 & 3 & 30 & 57 \\
\hline & $16: 22$ & $18^{\circ} 27^{\prime} 56^{\prime \prime} \mathrm{N} 69^{\circ} 55^{\prime} 07^{\prime \prime} \mathrm{W}$ & 2 & 2 & 31 & 64 \\
\hline & $16: 30$ & $18^{\circ} 27^{\prime} 57^{\prime \prime} \mathrm{N} 69^{\circ} 55^{\prime} 03^{\prime \prime} \mathrm{W}$ & 7 & 4 & 31 & 59 \\
\hline \multirow[t]{2}{*}{ Colón } & $15: 21$ & 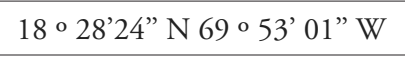 & 7 & 4 & 33 & 54 \\
\hline & $18: 47$ & $18^{\circ} 28^{\prime} 24^{\prime \prime} \mathrm{N} 69^{\circ} 53^{\prime} 01^{\prime \prime} \mathrm{W}$ & 8 & 8 & 29 & 64 \\
\hline \multirow[t]{2}{*}{ Mirador Sur } & $17: 11$ & $18^{\circ} 27^{\prime} 00^{\prime \prime} \mathrm{N} 69^{\circ} 56^{\prime} 20^{\prime \prime} \mathrm{W}$ & 4 & 2 & 29 & 56 \\
\hline & $17: 49$ & $18^{\circ} 26^{\prime} 31^{\prime \prime} \mathrm{N} 69^{\circ} 57^{\prime} 32^{\prime \prime} \mathrm{W}$ & 12 & 10 & 29 & 57 \\
\hline
\end{tabular}

Fuente: elaboración propia.

Un total de 10 géneros de hongos aeronavegantes pertenecientes a cinco familias fueron aislados en los parques inventariados (tabla 4).

50 | Ciencia, Ambiente y Clima 2020; 3(1, enero-junio): 47-54 


\section{Evaluación de las partículas en suspensión atmosférica y bioaerosoles fúngicos en parques urbanos de la ciudad}

de Santo Domingo, República Dominicana

Tabla 4. Hongos microscópicos presentes en parques urbanos Colón, Iberoamericano y Mirador Sur del Distrito Nacional de República Dominicana

\begin{tabular}{|c|c|c|c|c|c|}
\hline \multicolumn{2}{|c|}{ Género y/o especie } & Familias & Parque Colón & Parque Iberoamericano & Parque Mirador sur \\
\hline \multicolumn{2}{|c|}{ Alternaria cf. alternata } & Pleosporaceae & $\cdot$ & $\cdot$ & $\cdot$ \\
\hline \multicolumn{2}{|c|}{ Aspergillus niger } & Trichocomaceae & - & - & $\cdot$ \\
\hline \multicolumn{2}{|c|}{ Aspergillus sp. 1} & Trichocomaceae & $\bullet$ & $\bullet$ & $\bullet$ \\
\hline \multicolumn{2}{|c|}{ Cladosporium } & Davidiellaceae & • & $\bullet$ & • \\
\hline \multicolumn{2}{|c|}{ Dreschlera } & Pleosporaceae & $\cdot$ & & \\
\hline \multicolumn{2}{|c|}{ Fusarium sp. } & Nectriaceae & & & $\bullet$ \\
\hline \multicolumn{2}{|c|}{ Nattrasia sp. } & Botryosphaeriaceae & & & $\cdot$ \\
\hline \multicolumn{2}{|c|}{ Paecilomyces sp. } & Trichocomaceae & & $\cdot$ & $\cdot$ \\
\hline \multicolumn{2}{|c|}{ Penicillium sp. 1} & Trichocomaceae & • & $\bullet$ & $\bullet$ \\
\hline \multicolumn{2}{|c|}{ Penicillium sp. 2} & Trichocomaceae & & $\cdot$ & • \\
\hline \multicolumn{2}{|c|}{ Rhizophus sp. } & Mucoraceae & & & $\bullet$ \\
\hline \multicolumn{2}{|c|}{ Ulocladium sp. } & Pleosporaceae & & & • \\
\hline \multicolumn{6}{|c|}{ Leyenda } \\
\hline Presente & $\cdot$ & & & & \\
\hline Ausente & & & & & \\
\hline
\end{tabular}

Fuente: elaboración propia.

El valor máximo registrado del PM 2,5 $\mu \mathrm{m}$ fue de $16 \mu \mathrm{g} / \mathrm{Nm}^{3}$, lo que para la normativa de la República Dominicana, en cuanto al particulado en tiempo promedio de 24 horas, no supera el umbral estándar. Diversos autores han reportado que las concentraciones de este contaminante en un intervalo de 11 a $29.6 \mu \mathrm{g} / \mathrm{m}^{3}$, están relacionadas con morbilidad y mortalidad en ambientes urbanos (Blanco-Jiménez et al., 2012); además, que las partículas de suspensión atmosféricas (P.M) $2.5 \mu \mathrm{m}$ ocupan el noveno lugar en la lista de los factores de riesgo asociados con los índices de mortalidad a nivel mundial, principalmente por enfermedades cardiovasculares (López y Pérez, 2014; Suárez y Augusto, 2012).

La variación estacional del total de hongos en el aire y para las principales categorías genéricas (Alternaria, Aspergillus, Cladosporium y Penicillium) muestra una mayor concentración en el Parque Colón. Una de las razones puede ser la alta densidad de personas en el momento de la toma de muestras, junto a la presencia de palomas domésticas (Patagioenas leucocephala), las cuales representan una fuente de emisión de particulado de origen biológico (Suárez y Augusto, 2012).

Las categorías genéricas individuales siguieron el mismo patrón que el recuento total en el aire en su orden de distribución, fluctuación estacional y variación. Aspergillus y Penicillium fueron los géneros más predominantes, representando casi el $55 \%$ de los aislamientos totales, seguido de Cladosporium y Alternaria. Puede deberse al hecho de que Aspergillus, Penicillium y Cladosporium producen numerosas esporas pequeńas y ligeras, las cuales permanecen en el aire durante un largo período de tiempo, mientras que otros géneros de hongos producen menos esporas más grandes y pesadas que tienden a asentarse más rápidamente (Vonberg y Gastmeier, 2006). En varias investigaciones sobre esporas de hongos en el aire, se ha demostrado que los hongos más prevalentes pertenecían a los géneros Cladosporium, Alternaria, Curvularia, Aspergillus y Penicillium (Durugbo, Kajero, Omoregie y Oyejide, 
2013; Chakrabarti, Das y Gupta-Bhattacharya, 2012; Sepahvand, et al., 2011; Muhsin y Adlan, 2012; Lang-Yona et al., 2012).

De acuerdo con Dhanasekaran, Selvamani, Panneerselvam y Thajuddin (2009), la humedad relativa y el contenido de humedad de los materiales determinan en qué medida los diferentes microorganismos pueden crecer sobre materiales en ambientes interiores o exteriores. La mayor proporción de los bioaerosoles se encuentra cubierta por las esporas fúngicas, las cuales se ven influenciadas por factores biológicos y ambientales, entre ellos la temperatura y la humedad relativa. En varias investigaciones realizadas, los valores de temperatura y humedad relativa que dieron como resultado los patrones máximos de colonias de esporas fúngicas de bioaerosoles fueron $25-30{ }^{\circ} \mathrm{C}$, y $60-70 \%$ (Ramos y Meza, 2017). Por su parte, Pasanen, Lappalainen y Pasanen (1991) concluyeron que el tamaño de las esporas se ve relacionado a la humedad, es decir, a mayor humedad mayor tamańo. La humedad relativa promedio fue de $63 \%$ lo cual muestra una correlación positiva para las especies de Aspergillus y Penicillium aislados. Se necesitan realizar más monitoreos para determinar la concentración de esporas del género Cladosporium, la cual aumentó a medida que disminuyó la humedad relativa. Reyes, de la Cruz, Merino y Sánchez (2009) encontraron correlaciones positivas entre la humedad relativa con Cladosporium y Alternaria, estos patrones podrían ser los mismo, pero se necesitan más monitoreos para poderlo afirmar.

Para evaluar sistemáticamente la relación entre los hongos en el aire y los efectos adversos para la salud, es necesario conocer las concentraciones, los tipos y sus frecuencias relativas y diversidad de géneros y especies de hongos ambientales, no solamente en el parque Colón, sino también en otras áreas de la ciudad de Santo Domingo. Los géneros Alternaria y Cladosporium se consideran clínicamente los agentes alergénicos causales para la mayoría de los pacientes
(Dreborg et al., 1986). Estos géneros se encontraron presente en los tres parques muestreados (tabla 4).

\section{Conclusiones}

Esta investigación permitió determinar que los niveles del particulados $\leq 10 \mu \mathrm{m}$ e $\leq 2,5 \mu \mathrm{m}$ en los parques Mirador Sur, Iberoamericano y Colón, no superan el límite en tiempo promedio de 24 horas del Reglamento Técnico Ambiental de Calidad del Aire del Ministerio de Medio Ambiente y Recursos Naturales de la República Dominicana.

Las condiciones atmosféricas y el tipo de clima que presentaron los tres parques favorecen el desarrollo fúngico y la liberación de esporas fúngicas prevalecientes en el aire, entre ellas Alternaria y Cladosporium identificadas en los tres parques, consideras desde el aspecto clínico agentes alergénicos causales. Se necesita hacer más estudios para comprender la dinámica de los factores abióticos que influyen en la ecología de los hongos aeronavegantes.

\section{Agradecimientos}

Los autores quieren agradecer al Centro de Prevenciones y Control de Enfermedades Transmitidas por Vectores y Zoonosis y al Instituto Tecnológico de Santo Domingo (INTEC) por las facilidades prestadas en la utilización de los equipos de microscopia y laboratorio. A Ivelisse Montero de la Sociedad Dominicana de Micología por la revisión del manuscrito.

\section{Referencias}

Atlas of Airborne Fungal Spores in Europe (1993). En S. Nilsson, (Ed.) Berlin, Heidelberg: Springer-Verlag.

Baron, P. A. \& Willike, K. (2001). Aerosol Measurement - Principles, Techniques, and Applications, (2nd Ed.), John Wiley \& Sons. 
Blanco-Jiménez S., Altúzar F., Jiménez B., Aguilar G., Pablo M. \& Benítez M.A. (2015). Evaluación de Partículas Suspendidas PM2.5 en el Area Metropolitana de Monterrey. Instituto Nacional de Ecología y Cambio Climático (INECC). México.

Chakrabarti H. S., Das S., \& Gupta-Bhattacharya S. (2012). Outdoor airborne fungal spore load in a suburb of Kolkata, India: its variation, meteorological determinants and health impact. Int J Environ Health Res., 22, 37-50.

Dhanasekaran, D., Selvamani S., Panneerselvam, A., \& Thajuddin, N. (2009). Isolation and characterization of actinomycetes in Vellar Estuary, Annagkoil, Tamil Nadu. African Journal of Biotechnology, 8, 4159-4162.

Dreborg, S., Agrell, B., Foucard, N., Kjellman, M., Koivikko, A., \& Nilsson, S. (1986). A doubleblind, multicenter immunotherapy trial in children, using a purified and standardized Cladosporium herbarum Preparation I. Clinical Results. Allergy, 41, 131-140.

Durugbo E. U., Kajero A. O., Omoregie E. I. \& Oyejide N. E. (2013). A survey of outdoor and indoor airborne fungal spore in the Redemption City, Ogun State, south-western Nigeria. Aerobiologia, 29, 201-216.

Estrada, R., Alvarado, A., Gutiérrez, M., Salcedo, R. \& Moreno, S. (2013). Variación temporal de esporas de Alternaria, Cladosporium, Coprinus, Curvularia y Venturia en el aire del área metropolitana de Monterrey, Nuevo León, México. Revista Internacional de Contaminación Ambiental, 29(2): 155-165.

Gioulekas, D., Balafoutis, C., Damialis, C., Papakosta, D., Gioulekas, G. \& Patakas, D. (2004). Fifteen years' record of airborne allergenic pollen and meteorological parameters in Thessaloniki, Greece. International Journal of Biometeorology, $48,128-136$.

Gregory, P. H. (1973). The Microbiology of the Atmosphere. Leonard Hill, England.
Griffin, D. (2007). Atmospheric Movement of Microorganisms in Clouds of Desert Dust and Implications of Human Health. Clinical Microbiology Reviews, 20, 459 -477.

Hedayati, M.T., Pasqualotto, A.C., Warn, P.A., Bowyer, P. \& Denning, D.W. (2007). Aspergillus flavus: human pathogen, allergen and mycotoxin producer. Microbiology, 153, 16771692.

Jiménez-Orozco, C., Lebrón-Liriano, B. V., Fernández-Gutiérrez, R., Urbáez, R., \& Guerrero, Ángela. (2019). Caracterización de la flora epífita vascular del Parque Iberoamérica, Santo Domingo, República Dominicana. Ciencia, Ambiente y Clima, 2(1), 23-33. Doi: https:// doi.org/10.22206/cac.2019.v2i1.pp23-33

Karim, S. (2010). Estudio Microbiología Del Material Particulado Atmosférico De Santiago Mediante Herramientas De Biología Molecular. Tesis de postgrado. Universidad de Chile. Santiago de Chile. Disponible en http://mgpa.forestaluchile.cl/Tesis/Salvador\%20Barahona.pdf. [Recuperado el 08/08/2019].

Kendrick B. (2001). The Fifth Kingdom. Mycologue Publishing, Sydney, British Columbia.

Lacey J. (1991). Aerobiology and Health: The Role of Airborne Fungal Spores in Respiratory Disease, (pp. 157-185). In Frontiers in Mycology CAB International, Oxon, UK.

Lang-Yona N., Dannemiller K., Yamamoto N., Burshtein N., Peccia J., Yarden O. \& Rudich Y. (2012). Annual distribution of allergenic fungal spores in atmospheric particulate matter in the Eastern Mediterranean; a comparative study between ergosterol and quantitative PCR analysis. Atmos Chem Phys, 12, 2681-2690.

López, A. (2012). Evaluación bioaerosoles, bacterias, hongos, en el laboratorio de microbiología de la ESPE y construcción previa de un burbujeo experimental. (Tesis de grado). Escuela Politécnica del Ejército. Ecuador. Recuperado de https://repositorio.espe.edu.ec/bitstream/ 21000/5996/1/T-ESPE-034536.pdf 
López, M. T., \& Pérez, I. K. (2014). Valoración económica de los beneficios a la salud de la población que se alcanzarian por la reducción de las PM2.5 en tres zonas metropolitanas mexicanas. México: INECC.

Mitakakis, T., O'meara, T., \& Tovey, E. (2005). The effect of sunlight on allergen release from spores of the fungus Alternaria. Grana, 42, 43-6.

Montaño, M., \& Sandobal, S. (2007). Contaminación atmosférica y salud: Elementos ciencias y cultura. La Benemerita, 14, 29-33.

Muhsin T. M. \& Adlan M. M. (2012). Seasonal distribution pattern of outdoor airborne fungi in Basrah city, southern Iraq. J Basrah Res (Sciences), 38, 90-98.

Organización Mundial de la Salud. (2005). Guias de calidad del aire de la OMS relativas al material particulado, el ozono, el dióxido de nitrógeno y el dióxido de azufre: Resumen de valuación de riesgos. [Internet]. Disponible en https://apps. who.int/iris/bitstream/handle/10665/69478/ WHO_SDE_PHE_OEH_06.02_spa.pdf; jsessionid $=8305 \mathrm{BBBAA} 30 \mathrm{~A} 965598 \mathrm{EE}-$ 3C5A08B7A67F? sequence $=1$ [Recuperado el 07/08/2019].

Pasanen, A.L., Lappalainen, S., \& Pasanen, P. (1996). Volatile organic metabolites associated with some toxic fungi and their mycotoxins. Analyst, 121, 1949-1953.

Quijano, A., Quijano, M., \& Henao, J. (2010). Caracterización fisicoquímica del material particulado fracción respirable PM2.5 en Pamplona-Norte de Santander Colombia. Bistua. Revista de la Facultad de Ciencias Básicas, 8(1), 1-20.
Ramos, R., \& Meza, V. (2017). Efectos de algunos factores meteorológicos sobre la concentración de esporas de hongos en la Plaza San Martín de Lima. Ecología Aplicada, 16(2), 143-149.

Reyes, E.S., de la Cruz D. R., Merino E. S. \& Sánchez, J.S. (2009). Meteorological and agricultural effects on airborne Alternaria and Cladosporium spores and clinical aspects in Valladolid (Spain). Ann Agric Environ Med., 16, 53-61.

Samson, R. A., Hoekstra, E. S., \& Frisvad, J. C. (2004). Introduction to Food and Airborne Fungi. (7th Ed.). American Society for Microbiology. Utrech.

Sepahvand A., Shams-Ghahfarokhi M., Allameh A., Jahanshiri Z., Jamali M., \& Razzaghi-Abyaneh M. (2011). A survey on distribution and toxigenicity of Aspergillus flavus from indoor and outdoor hospital environments. Folia Microbiol, 56, 527-534.

Suárez, A., \& Augusto, C. (2012). Diagnóstico y control de materia particulado: Partículas suspendidas totales y fracción respirable PM10. Revista Luna Azul, 34, 195-213.

USAID-ICMA-FEDOMU-ADN. (2017). Diagnóstico Territorial del Distrito Nacional. (7). [Internet]. Disponible en http://adn.gob. do/pot/2-DOCUMENTOS-BASE/2.5[DN]-Diagnostico-Territorial.pdf) [Recuperado el 08/05/2020]

Vonberg R. P. \& Gastmeier, P. (2006). Nosocomial aspergillosis in outbreak settings. J. Hosp Infect, 63, 246-254. 